\title{
Numerical study of magnetic nanofluids flow in the round channel located in the constant magnetic field
}

\author{
Maxim Pryazhnikov ${ }^{1,2, *}$, Dmitriy Guzei ${ }^{1}$, Andrey Minakov ${ }^{1,2}$ and Tatyana Rodionova ${ }^{1}$ \\ ${ }^{1}$ Siberian Federal University, 79 Svobodny pr., Krasnoyarsk, 660041, Russia \\ ${ }^{2}$ Institute of Thermophysics SB RAS, 1 Acad. Lavrentiev pr., Novosibirsk, 630090, Russia
}

\begin{abstract}
In this paper, the study of ferromagnetic nanoparticles behaviour in the constant magnetic field is carried out. For numerical simulation we have used Euler-Lagrange two-component approach. Using numerical simulation we have studied the growth of deposition of nanoparticles on the channel walls depending on the Reynolds number and the position of the magnet. The flow pattern, the concentration field and the trajectory of nanoparticles as a function of the Reynolds number were obtained. The good qualitative and quantitative agreement between numerical simulation and experiments was shown.
\end{abstract}

\section{Introduction}

One of the promising directions in the treatment of cancer may be the use of magnetic nanoparticles [1-3]. The essence of this approach is that the magnetic nanoparticles of sizes of 5-100 $\mathrm{nm}$ are injected into the bloodstream and concentrated in the tumor by an external magnetic field. Further, nanoparticles are heated in the tumor either by laser radiation or electromagnetic alternating fields. This leads to the death of cancer cells. The minimum harm is caused to healthy cells. At present, the effectiveness of this approach has been confirmed by many experiments on animals and humans during the treatment of cancer. There are lots of works study of nanoparticles transport in the magnetic field in the channels [1-4]. There are so much unresolved problems and issues related to transfer mechanisms, concentration and heating of nanoparticles in fluids and tissues, and their control under the influence of external electromagnetic fields.

In this paper, we conducted the numerical simulation of ferromagnetic nanoparticles behavior flowing in the liquid in the round pipe in the constant magnetic field.

Detailed description of the experimental study is presented in the paper [5]. The round channel is a glass tube with the inner diameter of $6 \mathrm{~mm}$ and the length of $50 \mathrm{~cm}$. The tube is connected with a pump and a container with liquid. We used gear pump WT30001JA to

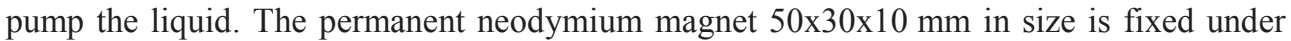
the glass tube. Magnetic induction of the constant magnetic field was measured by

\footnotetext{
* Corresponding author: arrivent@yandex.ru
} 
milliteslameter TPU. Distilled water with the nanoparticles was pumping through the tube. The particles interacted with the magnetic field and deposited on the tube wall.

Investigation of particles deposition process was conducted in the ferromagnetic fluid based on distilled water contained the nanoparticles $\mathrm{Fe}_{3} \mathrm{O}_{4}$. Volumetric concentration of particles was very low $\left(10^{-4} \%\right)$. The size of the nanoparticles was $50 \mathrm{~nm}$.

\section{Mathematical model}

The numerical simulation of nanoparticles transport in the constant magnetic field has been carried out. We used two-component model of nanofluids to describe the motion of nanoparticles in the magnetic field. This model considers the nanofluids as a binary mixture of base fluid and nanoparticles. The equations of this model are as follows:

$$
\begin{gathered}
\frac{\partial \rho}{\partial t}+\nabla \cdot(\rho \mathbf{v})=0 \\
\frac{\partial \rho \mathbf{v}}{\partial t}+\nabla \cdot(\rho \mathbf{v})=-\nabla p+\nabla \cdot \mathbf{T}+\mathbf{F}_{\mathrm{m}} \\
\frac{\partial \rho C_{\mathrm{m}}}{\partial t}+\nabla \cdot\left(\rho \mathbf{v}_{\mathrm{p}} C_{\mathrm{m}}\right)=\nabla \cdot\left(\rho D C_{\mathrm{m}}\right) \\
\mathbf{T}=\mu \cdot\left(\nabla \mathbf{v}+\nabla \mathbf{v}^{T}-\frac{2}{3} \nabla \cdot \mathbf{v E}\right)
\end{gathered}
$$

where $\rho$ - density of nanofluid, $\mathbf{v}$ - velocity vector, $p$ - pressure, $\mathbf{T}-$ viscous stress tensor, $\mathbf{F}_{\mathrm{m}}$ - electromagnetic force, $C_{\mathrm{m}}-$ mass flow rate of nanoparticles, $\mathbf{v}_{\mathrm{p}}$ - particle velocity, $D-$ diffusion coefficient, $\mu$-dynamic viscosity, $\mathbf{E}$ - single tensor.

Volumetric electromagnetic force is found using the following formula:

$$
\mathbf{F}_{m}=\varphi \cdot \mu_{0} \frac{3 \chi}{\chi+3}(\mathbf{H} \cdot \nabla) \mathbf{H}
$$

where $\varphi$ - volume concentration, $\mu_{0}$ - magnetic constant, $\chi$ - magnetic susceptibility of the material, $\mathbf{H}$ - magnetic field.

The particle velocity is calculated using the following formula:

$$
\mathbf{V}_{p}=\mathbf{v}+\frac{\mathbf{F}_{m} \cdot V_{\mathrm{d}}}{\varphi \cdot 6 \pi \mu r_{\mathrm{p}}}
$$

where $V_{\mathrm{d}}$ - volume of the particle, $r_{\mathrm{p}}$ - radius of the particle.

Density and heat capacity are computed as follows:

$$
\begin{gathered}
\rho=\varphi \rho_{\mathrm{p}}+(1-\varphi) \rho_{\mathrm{w}} \\
C_{\mathrm{p}}=\frac{\varphi \rho_{\mathrm{p}} C_{\mathrm{p}, \mathrm{p}}+(1-\varphi) \rho_{\mathrm{w}} C_{\mathrm{p}, \mathrm{w}}}{\rho}
\end{gathered}
$$

We have to add equations of magnetostatics to obtain a closed system of equations.

Component of the magnetic field around the magnet can be found by the magnetic field vector potential: 


$$
\mathbf{B}=\nabla \times \mathbf{A}
$$
follows:

The equation of the magnetic field vector potential in the absence of vortex is as

$$
\nabla \times\left(\frac{1}{\mu_{0}}\left(\nabla \times \mathbf{A}-\mathbf{B}_{\mathrm{R}}\right)\right)=0
$$

where $\mathbf{B}_{\mathrm{R}}$ - residual magnetic induction which is equal to zero everywhere except the region occupied by the magnet.

The magnetic field intensity can be found using the following formula:

$$
\mathbf{H}=\mathbf{B} / \mu_{0}
$$

\section{Numerical results}

The problem statement was the same as experimental study [5]. The computational domain was represented as the round channel with the inner diameter of $6 \mathrm{~mm}$ and the length of $50 \mathrm{~cm}$. Computational mesh consisted of half a million nodes.

Numerical simulation of nanoparticles transport in the constant magnetic field are carried out for various Reynolds numbers. The flow patterns in channel without a magnetic field are shown in figures 1-2. As you can see nanoparticles move strictly along the streamlines. They do not deposit on the wall of the channel. Velocity vector field in the channel and trajectory of nanoparticles are shown in figures 3-4. Large deposits of particles are formed on the channel wall. The numerical results correlate with the experimental data. This can be seen in figure 5. You can also see that the interaction of the magnetic field with the particles flow leads to formation of the flow recirculation zones. The deposits are formed in the flow recirculation zones. In this case nanoparticles move like it is shown in figure 4. It shows that at low flow rate all the nanoparticles deposit on the channel walls.

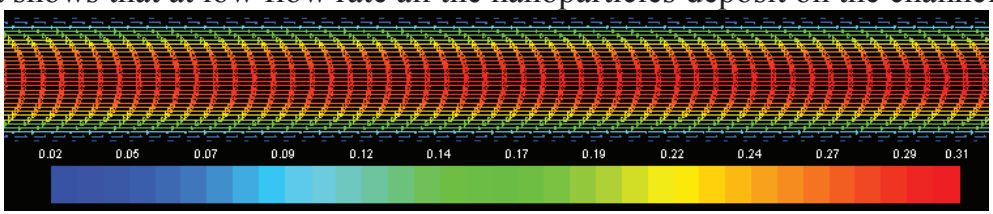

Fig. 1. Velocity vector field in the channel at $\mathrm{Re}=1200$. Without the magnetic field.

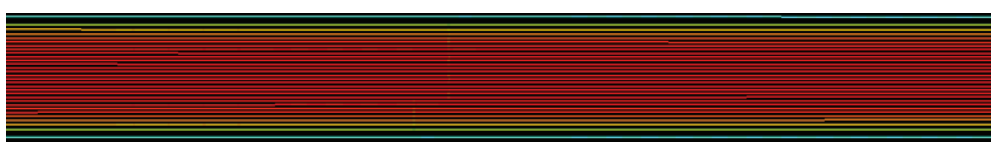

Fig. 2. Velocity vector field in the channel at $\mathrm{Re}=1200$. Without the magnetic field.

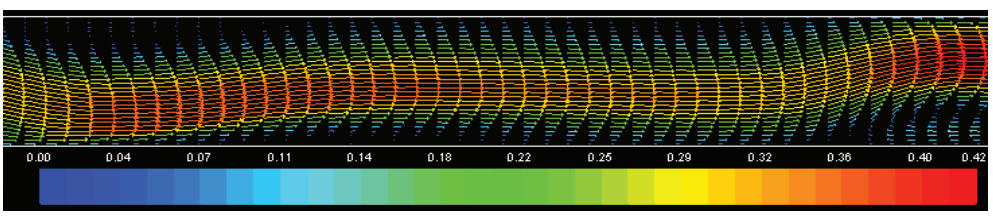

Fig. 3. Velocity vector field in the channel at $\mathrm{Re}=1200$. 


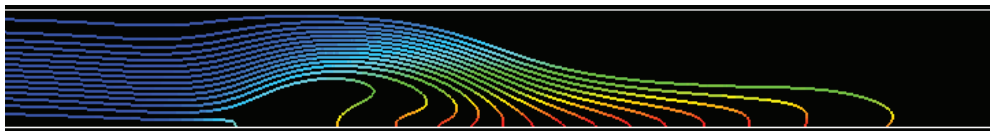

Fig. 4. Trajectory of nanoparticles at $\mathrm{Re}=1200$.

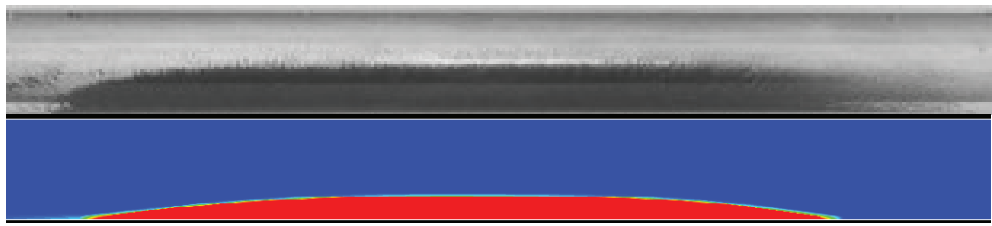

Fig. 5. Comparison of particle deposition shape in the channel at $\mathrm{Re}=1200$ (top - experimental photo, bottom - simulation)

The higher the Reynolds number, the more complicated the flow. The shape of the vortex region has changed near the channel wall (see fig. 6). The deposits form has also changed. However, the numerical simulation reproduces perfectly the shape of deposition obtained during the experiment.

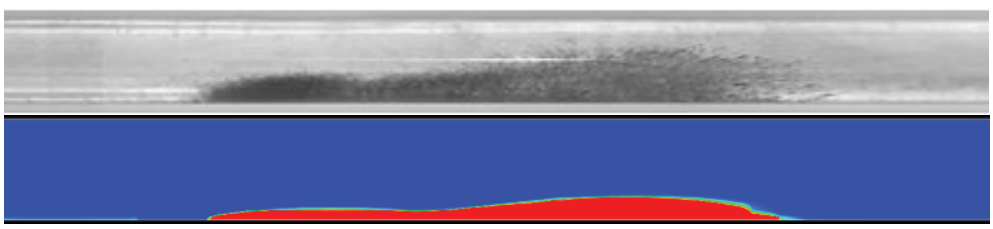

Fig. 6. Comparison of particle deposition shape in the channel at $\mathrm{Re}=2810$ (top - experimental photo, bottom - simulation)

\section{Conclusion}

The study of ferromagnetic nanoparticles behaviour flowing in the round channel with the liquid in the constant magnetic field was carried out. To simulate the transport of nanoparticles in the magnetic field a novel Euler-Lagrange combined two-component approach was used. The verification and adaptation of mathematical models of magnetic nanoparticles transport under the influence of external electromagnetic fields based on the experimental data was performed. The numerical results were compared with experimental data. Good qualitative and quantitative agreement is obtained between numerical simulation and experiment. In future it is planned to measure the velocity profiles for these regimes using particle image velocimetry (PIV) method.

The reported study was funded by Russian Foundation for Basic Research, Government of Krasnoyarsk Territory, Krasnoyarsk Region Science and Technology Support Fund to the research project №16-48-243061.

\section{References}

1. J. Dobson, Drug Dev. Res. 67 (2006)

2. P.A. Voltairas, D.I. Fotiadis, L.K. Michalis, J. Biomech. 35 (2002)

3. H.D. Liu, W. Xu, S.G. Wang, Z.J. Ke, Appl. Math. Mech.-Engl. Ed. 29 (2008)

4. C. M. Oldenburg, S. E. Borglin, G. J. Moridis, Transport in Porous Media 38 (2000)

5. M. Pryazhnikov, D. Guzei, A. Minakov, V. Zhigarev, A. Shebeleva, MATEC Web of Conferences 72 (2016) 